\title{
Björn Technau. 2018. Beleidigungswörter. Die Semantik und Pragmatik pejorativer Personenbezeichnungen (Linguistik - Impulse und Tendenzen 74). Berlin, Boston: De Gruyter. 377 S.
}

Besprochen von Sebastian Veletić: TU Dresden, Professur für germanistische Linguistik und Sprachgeschichte, Wiener Straße 48, D-01062 Dresden,

E-Mail: sebastian.veletic@tu-dresden.de

https://doi.org/10.1515/zrs-2019-2021

Beleidigungswörter gehören aufgrund ihrer abwertenden Bedeutung zu den Tabuwörtern, dennoch erfreuen sie sich insbesondere in der gesprochenen Sprache einer gesellschaftlich weit verbreiteten Verwendung. Dadurch stoßen sie nicht nur in der Öffentlichkeit, sondern auch in der Wissenschaft auf großes Interesse und werden aus verschiedenen Forschungsperspektiven kontrovers diskutiert. Björn Technau widmet sich in seiner Monographie insbesondere den Beleidigungswörtern, die auf bestimmte Personengruppen bezogen sind, und intendiert dabei eine Aufschlüsselung der semantischen und pragmatischen Bedeutungsaspekte von Beleidigungswörtern, um so einen Beitrag zur Erforschung der Pejorativa und zur Semantik/Pragmatik-Forschung zu leisten.

Technau führt zunächst in die unterschiedlichen Arten pejorativer Sprache ein, gibt einen Überblick über die Semantik/Pragmatik-Schnittstelle, an welcher er Beleidigungswörter theoretisch verortet, und legt seine Untersuchungsmethode dar (Kap. 1). Darauf aufbauend widmet sich Technau anhand eines selbst entwickelten semantischen Analysemodells den Bedeutungskomponenten von Beleidigungswörtern und setzt sich darüber hinaus mit den Quellen des Beleidigungsgrades und dem Bedeutungswandel von Beleidigungswörtern auseinander (Kap. 2). Das darauffolgende Kapitel zur Pragmatik der Pejorativa nimmt die Verwendungsweisen von Beleidigungswörtern in den Blick (Kap. 3). Abschließend fasst Technau die Ergebnisse zusammen und gibt einen Forschungsausblick (Kap. 4).

\section{Forschungsgegenstand}

Zur genauen Abgrenzung des Forschungsgegenstands entwickelt Technau ein umfangreiches Kategorisierungssystem für verbale und nonverbale tabuisierte Kommunikationsmittel, in dem auch die verschiedenen Arten von Beleidigungswörtern, d.h. „nominale Lexeme, zumeist Personenbezeichnungen, mit pejorativer Bedeutung“ (S. 3), verortet sind. Technau unterscheidet zwischen vier Arten 
von Beleidigungswörtern in Abhängigkeit von den beiden Kriterien, ob die Beleidigungswörter auf eine bestimmte Personengruppe (im Folgenden: PG) referieren und ob sie ein nicht-pejoratives Korrelat (im Folgenden: NPC) aufweisen (vgl. S. 5-7). So gelingt es ihm, die vielfältigen Erscheinungsformen pejorativer Sprache zu identifizieren und terminologisch voneinander abzugrenzen. Im Zentrum der Untersuchung stehen Beleidigungswörter ${ }^{+\mathrm{PG}+\mathrm{NPC}}$, also Beleidigungswörter, die pejorativ auf eine PG verweisen und ein nicht-pejoratives Gegenstück haben (vgl. S. 8). ${ }^{1}$

\section{Methode und Hypothesen}

Auf Grundlage der einschlägigen Bedeutungstheorien entwickelt Technau konzeptuell-analytisch ein vielschichtiges Analysemodell, das sog. Multikomponentenmodell, durch welches die konstatierten Defizite bisheriger Ansätze ausgeräumt werden (vgl. Kap. 2.1). Mithilfe einer Fragebogenuntersuchung werden das konzipierte Analysemodell sowie drei Hypothesen zur Semantik von Beleidigungswörtern empirisch überprüft. Die Hypothesen stellen darauf ab, dass die semantischen Bedeutungsaspekte konventionalisiert sind, d.h., dass es in der Sprachgemeinschaft einen Konsens darüber gibt, (1) auf welche Personengruppen Beleidigungswörter ${ }^{\mathrm{PG}}$ verweisen und (2) wie hoch der Beleidigungsgrad von Beleidigungswörtern $^{\mathrm{PG}}$ und Beleidigungswörtern ${ }^{-\mathrm{PG}-\mathrm{NPC}}$ ist (vgl. Kap. 1.2.1). Die zentrale Frage, die der Identifizierung der semantischen Bedeutungskomponenten dient, lautet: „Was muss ein Sprecher über ein Beleidigungswort ${ }^{\mathrm{PG}}$ wissen [...], um es kompetent einsetzen und verstehen zu können?“ (S. 69). Die dritte anhand des Fragebogens geprüfte Hypothese besagt, (3) dass die Adjektive schwul und behindert konventionalisiert für negativ bewertete Situationen Verwendung finden (vgl. S. 28). Ein wesentlicher Vorzug dieser empirischen Herangehensweise ist, dass die Untersuchung durch den Blick auf das Sprachwissen und den Sprachgebrauch - im Vergleich zu einigen früheren Studien - an der Realität der Sprachgemeinschaft orientiert ist.

Ferner soll eine Konversationsanalyse Aufschluss über die Pragmatik von Beleidigungswörtern geben. Als Untersuchungsmaterial werden dafür neben schriftlichen Belegen aus Webforen und sozialen Medien insbesondere aufgezeichnete informelle mündliche Gespräche langjährig miteinander befreundeter Personen herangezogen (vgl. Kap. 1.2.2).

1 In Analogie zur Terminologie von Technau werden die Beleidigungswörter ${ }^{+\mathrm{PG}+\mathrm{NPC}}$ im Folgenden verkürzt als Beleidigungswörter ${ }^{\mathrm{PG}}$ bezeichnet (vgl. S. 8). 


\section{Semantik der Pejorativa}

Die sehr präzise, jedoch nicht zu detaillierte Auswertung der Fragebogenergebnisse mündet in eine plausible Aufschlüsselung der Bedeutungskomponenten von Beleidigungswörtern ${ }^{\mathrm{PG}}$. Die einzelnen semantischen Komponenten werden nacheinander unter Rückbezug auf die vorgestellten theoretischen Ansätze und unter Einbezug der empirischen Daten transparent vorgestellt und nachvollziehbar diskutiert (vgl. Kap. 2.2). Technau unterscheidet im Hinblick auf den semantischen Gehalt von Beleidigungswörtern ${ }^{\mathrm{PG}}$ zwischen vier Bedeutungskomponenten: (1) die referentielle, die die Extension des Beleidigungswortes ${ }^{\mathrm{PG}}$ bestimmt und dem NPC entspricht, (2) die pejorative, die diese Extension abwertet, (3) die expressive, die indexikalisch die gesteigerten Emotionen im Verwendungskontext anzeigt, und (4) die skalare, die auf den individuellen Beleidigungsgrad verweist (vgl. S. 337). Die Existenz einer referentiellen und einer skalaren Bedeutungskomponente wird empirisch dadurch nachgewiesen, dass sich unter den Fragebogenteilnehmer ${ }^{\star}$ innen bei der Mehrheit der getesteten Beleidigungswörter ein Konsens hinsichtlich der bezeichneten PG und des Beleidigungsgrades feststellen lässt (vgl. Kap. 2.2.1 und Kap. 2.2.3). Die pejorative und die expressive Bedeutungskomponente werden nicht empirisch geprüft, jedoch überzeugend theoretisch untermauert und plausibel erweitert. Dadurch, dass die expressive Bedeutungskomponente bspw. so definiert ist, dass sie sowohl auf negative als auch auf positive Emotionen verweisen kann, bietet sie einen Erklärungsansatz für die verschiedensten Verwendungsweisen von Beleidigungswörtern zwischen Scherzkommunikation und Hate Speech (vgl. S. 138-142). Zudem hebt sich die skalare Bedeutungskomponente im Multikomponentenmodell insofern von bisherigen Beschreibungsansätzen ab, als sie sich aus einer Vielzahl von Quellen speist, die in semantische, pragmatische und außersprachliche Quellen unterteilt werden, wodurch erklärt werden kann, warum Beleidigungswörter, die auf dieselbe PG referieren, unterschiedlich stark beleidigend sind, z.B. Neger - Nigger (vgl. S. 106-107). Technau weist aber mehrfach darauf hin, dass Beleidigungswörter eine differenzierte und individuelle Analyse verlangen, „da sich nicht für alle gleichermaßen ein Konsens bzgl. der Bedeutungskomponenten abzeichnet“ (S. 209).

An die Beschreibung des Multikomponentenmodells schließt Technau ein Kapitel zu den Quellen des Beleidigungsgrades an, die die Sprecher*innen über den Beleidigungsgrad von Beleidigungswörtern informieren. Darin diskutiert Technau anhand von zahlreichen anschaulichen Beispielen in erster Linie die Diskriminierungspraxis und die Stereotype als außersprachliche Quellen sowie die Sprachempfehlungen im Sinne der Political Correctness, Gerichtsurteile und die Medien als pragmatische Quellen; die semantischen Quellen werden hingegen nur am Rande beleuchtet (vgl. Kap. 2.3). Warum der Fokus in Anbetracht 
der Vielfalt möglicher Quellen auf die genannten gelegt wird, bleibt unbegründet. Kritisch sei an dieser Stelle außerdem angemerkt, dass einige Quellen des Beleidigungsgrades einer empirischen Überprüfung bedürften: Die Aussage etwa, dass die öffentliche Diskussion über Beleidigungen potentiell einen Einfluss auf den Beleidigungsgrad des jeweiligen Beleidigungswortes hat, erscheint in Anbetracht der fehlenden empirischen Grundlage etwas spekulativ (vgl. S. 180).

Das darauffolgende Kapitel ist dem Bedeutungswandel von Beleidigungswörtern gewidmet. So können etwa neutrale Wörter zu Beleidigungswörtern werden (z.B. Weib und Neger) und umgekehrt (z.B. gay und queer) (vgl. Kap. 2.4). Ein allmählicher Bedeutungswandel bedingt durch den Verlust der referentiellen Komponente zeichne sich bspw. bei den Adjektiven behindert und schwul ab, die inzwischen zur Benennung negativer Situationen konventionalisiert seien und ohne einen Bezug zur PG der behinderten bzw. homosexuellen Personen Verwendung fänden (vgl. S. 212). Allerdings ist der prognostizierte Wandel des Beleidigungswortes Schwuchtel hin zu einem Beleidigungswort ${ }^{-P G-N P C}$, das zur allgemeinen Abwertung von bestimmten Eigenschaften wie Schwäche und Empfindlichkeit und ohne einen abwertenden Bezug auf die PG der homosexuellen Männer verwendet werde, fragwürdig, da die Verwendungsweisen eines Rappers und seiner Community sowie seine metasprachlichen Reflexionen, die als Beleg für den Bedeutungswandel herangezogen werden, nicht hinreichend sind, um von einer „Regelmäßigkeit solcher Verwendungsweisen“ (S. 206) sprechen zu können. Ebenso können Verwendungsweisen und Bedeutungswandelprozesse englischer Beleidigungswörter (z.B. faggot) nicht auf übersetzungsäquivalente deutsche Beleidigungswörter (z.B. Schwuchtel) transferiert werden (vgl. S. 204206).

\section{Pragmatik der Pejorativa}

Bevor Technau die Verwendungsweisen von Beleidigungswörtern ${ }^{\mathrm{PG}}$ einer Konversationsanalyse unterzieht, bettet er zunächst die Verwendung von Pejorativa in kommunikationstheoretische Ansätze ein, indem er den Zusammenhang zwischen Beleidigungswörtern und gesprochener Sprache, den Interaktionsmodalitäten, dem Grice'schen Kooperationsprinzip, (Un)Höflichkeit sowie dem faceKonzept beleuchtet (vgl. Kap. 3.1). Der pragmatischen Analyse sind außerdem Reflexionen über die Sprecher*innen und die mit der Verwendung von Beleidigungswörtern verbundenen Emotionen und Aggressionen vorangestellt (vgl. Kap. 3.2 und 3.3). Unter Rückgriff auf eine Vielzahl von Studien stellt Technau fest, dass die Verwendung von Beleidigungswörtern für diverse soziale Gruppen dokumentiert ist und weniger mit Geschlecht und Alter als vielmehr mit Per- 
sönlichkeitsmerkmalen und dem sozialen Umfeld zusammenhängt (vgl. S. 230233). So werden Beleidigungswörter auch in der untersuchten Sprachgemeinschaft verwendet, jedoch nicht zur sprachlichen Abwertung von Personen(gruppen); vielmehr werden mit Beleidigungswörtern wie Jakobswegscheißerin, white trash, verweichlichter Westassi und verwöhntes Balg u.a. humoristische Effekte intendiert (vgl. S. 240-246).

Im Wesentlichen unterscheidet Technau demnach zwischen drei Verwendungsweisen der Beleidigungswörter, nämlich einer pejorativen (z.B. in Hate Speech), einer nicht-pejorativen (z.B. bei Appropriation) und einer neutralen (z.B. in metasprachlichen Reflexionen) (vgl. S. 266). In diesem Zusammenhang stellt er heraus, dass nicht-pejorative Verwendungsweisen weitaus häufiger sind als pejorative und dass beide Verwendungsweisen „hauptsächlich in Abwesenheit der Target Group stattfinden“ (S. 284). Mit einem äußerst differenzierten Blick untersucht Technau die Verwendung verschiedenster Beleidigungswörter in unterschiedlichen Kontexten. Dabei räumt er ein, dass sich einige Verwendungsweisen einer eindeutigen Kategorisierung entziehen, so z.B. Banter, das als interaktionales scherzhaftes Phänomen mit potentiell beleidigendem Effekt ,auf einem Kontinuum zwischen sozialer Bindung und Abgrenzung“ (S. 334) zu verorten ist und daher auch nicht eindeutig den nicht-pejorativen Verwendungsweisen zugeordnet werden kann. Auf ähnliche Kategorisierungsprobleme stößt auch die Appropriation, d.h. „[d]ie Aneignung von Beleidigungswörtern ${ }^{\mathrm{PG}} \mathrm{zu}$ nicht-pejorativen Zwecken durch Mitglieder der bezeichneten PG“ (S. 315). Eine weitreichende Erkenntnis in Bezug auf die Appropriation ist, dass auch Nicht-Mitglieder der bezeichneten PG (Target Group) Beleidigungswörter ${ }^{\mathrm{PG}}$ wie Schwuchtel oder Neger in beschränkten sozialen Kontexten erfolgreich nicht-pejorativ verwenden können (vgl. S. 318). Daraus schließt Technau, dass der Erfolg nicht-pejorativer Verwendungsweisen keine Target-Group-Mitgliedschaft voraussetzt, sondern vielmehr von einer Ingroup-Mitgliedschaft abhängt, in der eine gemeinsame Gruppenidentität vorliegt, und zwar unabhängig von Hautfarbe, Religion oder sexueller Orientierung der Sprecher^innen (vgl. S. 320). Um bspw. Schwuchtel erfolgreich nichtpejorativ verwenden zu können, müsse man nicht zwingend der PG der homosexuellen Männer angehören; die notwendige Bedingung für den Erfolg einer nicht-pejorativen Verwendungsweise seien soziale Faktoren wie freundschaftliche Bande und ein geteiltes Wissen um die Einstellungen der Interaktionspartner*innen (vgl. S. 325). Es wird jedoch betont, dass auch bei prinzipiell nicht-pejorativen Verwendungsweisen eine latente Kritik mitschwingen kann, was die Kategorisierung wiederum erschwert (vgl. S. 322-323). Technau spürt somit bisher nicht beschriebene nicht-pejorative Zweifelsfälle auf und macht darauf aufmerksam, dass die „bloß binäre pejorativ/nicht-pejorativ-Unterscheidung“ (S. 327) die komplexe sprachliche Realität der Beleidigungswörter nicht abbilden kann. 


\section{Fazit}

Resümierend lässt sich festhalten, dass es Technau mit seiner Monographie gelingt, das weitgehend unerforschte Feld der Semantik und Pragmatik der deutschen Beleidigungswörter zu erschließen. Anhand von bedeutungs- und kommunikationstheoretischen Ansätzen zur pejorativen Sprache gibt er einen umfassenden Überblick über die bisherigen semantisch-pragmatischen Forschungsansätze und erfasst in sehr einleuchtender, differenzierter und transparenter Form den semantischen Gehalt und die Verwendungsweisen von Beleidigungswörtern ${ }^{\mathrm{PG}}$. Das im Rahmen der Arbeit entwickelte und empirisch erprobte semantische Analysemodell stellt zudem eine fundierte Grundlage für weitere Untersuchungen dar, was die Monographie anschlussfähig macht.

Trotz der Komplexität des Gegenstands büßt die Arbeit nicht an Lesbarkeit ein. Die durchgehend verwendete Ich-Form mag zunächst etwas ungewöhnlich und unsachlich anmuten, ist aber meines Erachtens eine erfrischende und elegante Alternative zum unpersönlichen Schreiben, die im Einklang mit der Modernität und Dynamik des Untersuchungsgegenstands steht. Des Weiteren erzielen die untersuchten Gesprächsausschnitte ihre humoristischen Effekte nicht nur bei den Gesprächsteilnehmer^innen, sondern auch - wie ich vermute - bei der Leser`innenschaft, was die Monographie zu einer willkommenen, unterhaltsamen Abwechslung macht. Abschließend lässt sich sagen, dass Technau - abgesehen von den empirisch unzureichend abgesicherten Aussagen zum Beleidigungsgrad und Bedeutungswandel bestimmter Beleidigungswörter - eine Monographie vorlegt, die durch einen differenzierten Blick und methodische Innovativität zu gewinnbringenden, interessanten Erkenntnissen gelangt und damit einen wertvollen Beitrag zur Erforschung der Semantik und Pragmatik der Beleidigungswörter leistet. 